\title{
Knockdown of circ-PVT1 inhibits the progression of lung adenocarcinoma and enhances the sensitivity to cisplatin via the miR-429/FOXK1 signaling axis
}

\author{
LIMIN CAO $^{1}, \mathrm{XUEFENG} \mathrm{ZHOU}^{2}, \mathrm{XI} \mathrm{DING}^{3}$ and DONGYUN GAO ${ }^{2}$ \\ ${ }^{1}$ Department of Respiratory Medicine, The Second People's Hospital of Lianyungang, Lianyungang, Jiangsu 222023; \\ Departments of ${ }^{2}$ Oncology and ${ }^{3}$ Pharmacy, The Affiliated Hospital of Nantong University, \\ Dongtai, Jiangsu 224200, P.R. China
}

Received January 25, 2021; Accepted April 20, 2021

DOI: $10.3892 / \mathrm{mmr} .2021 .12323$

\begin{abstract}
Lung cancer is one of the most prevalent cancers in China, and its incidence and morbidity remain high due to various independent factors. Lung adenocarcinoma (ADC) is the most common type of non-small cell lung carcinoma. Circular RNA plasmacytoma variant translocation 1 (circ-PVT1) plays an oncogenic role in various types of cancer, but the specific role of circ-PVT1 in lung ADC has not yet been reported. In the present study, circ-PVT1 was knocked down in A549 cells and the cell viability, proliferation, migration and invasion were measured via MTT, colony formation, wound healing and Transwell assays, respectively. Then, the cell viability of A549 cells with circ-PVT1-knockdown or -overexpression was detected after exposure to cisplatin (DDP). After confirming the associations among circ-PVT1, microRNA (miR)-429 and forkhead box k1 (FOXK1) using various tools and assays, the cellular functions of A549 cells treated with combined short hairpin (sh)RNA-circ-PVT1 and miR-429 inhibitor/pcDNA3.1-FOXK1 were tested again. The expression of circ-PVT1 was found to be increased in lung ADC cells, and shRNA-circ-PVT1 led to decreased cell viability, proliferation, migration and invasion. The expression of circ-PVT1 was higher in A549/DDP cells than that in A549 cells, and the activity of caspase-3 was also activated by DDP in A549/DDP cells transfected with shRNA-circ-PVT1, whereas it was inactivated by DDP in A549 cells transfected with circ-PVT1 overexpression plasmid. Furthermore, the decreased cell viability, proliferation, invasion and migration induced by shRNA-circ-PVT1 could be abated by transfection with miR-429 inhibitor and pcDNA3.1-FOXK1. In conclusion,
\end{abstract}

Correspondence to: Dr Dongyun Gao, Department of Oncology, The Affiliated Hospital of Nantong University, 2 Kangfu West Road, Dongtai, Jiangsu 224200, P.R. China

E-mail: gaodongyungdy@163.com

Key words: circRNA plasmacytoma variant translocation 1, lung adenocarcinoma, cisplatin, microRNA-429, forkhead box k1 interference of circ-PVT1 inhibits the progression of lung ADC and enhances its sensitivity to DDP via miR-429/FOXK1, which may provide a theoretical basis for the use of novel targets in the treatment of lung ADC.

\section{Introduction}

Lung cancer is one of the most prevalent types of cancer in China, and its incidence and morbidity remain high due to various independent factors, such as smoking, atmospheric pollution, cooking oil fume and chronic neonatal lung disease $(1,2)$. Lung cancer can be histologically classified into small cell lung carcinoma and non-small cell lung carcinoma (NSCLC), and $\sim 85 \%$ of patients with lung cancer are diagnosed with NSCLC (3). NSCLC can be further categorized into squamous-cell carcinoma, lung adenocarcinoma (ADC) and large-cell carcinoma. Among these subtypes, lung ADC is the most common type of NSCLC (4). Despite advances being made in the treatment methods available for NSCLC, the 5-year survival rate of patients has not improved and remains at $<15 \%$ (5).

Circular RNAs (circRNAs/circ) are newly identified non-coding RNAs (ncRNAs) that are present in the cytoplasm of eukaryotic organisms (6). circRNAs have been reported as potential molecular diagnosis markers in multiple types of cancer, including gastric cancer, oral squamous cell carcinoma and breast cancer $(7,8)$. A previous study reported that circRNA plasmacytoma variant translocation 1 (circ-PVT1) played an oncogenic role in gastric cancer by upregulating zinc finger E-box binding homeobox 1 expression via sponging microRNA (miRNA/miR)-124-3p (7). In addition, circ-PVT1 was found to increase the proliferation of epithelial ovarian cancer cells, and suppress apoptosis, via sponging miR-149, which promoted the progression of ovarian cancer (9). However, to the best of our knowledge, the specific role of circ-PVT1 in lung ADC has not yet been reported to date. As circRNAs can sponge miRNAs to regulate gene expression (10), the present study hypothesized that circ-PVT1 may be involved in the development of lung ADC by sponging certain miRNAs.

The association between circ-PVT1 and miR-429 was predicted using ENCORI in the present study. A previous study showed that the expression levels of miR-429 are 
downregulated in the serum of patients with NSCLC, and the decreased expression levels are associated with poor overall survival rate (11). Thus, miR-429 was suggested to be an independent risk factor for the prognosis of these patients. A previous study reported that the upregulated expression of long non-coding RNA metastasis-associated lung adenocarcinoma transcript 1 in human ADC cells and tissues was associated with tumor size, tumor node metastasis (TNM) stage and lymph node metastasis, and its expression levels were negatively correlated with miR-429 expression levels (12). However, other previous studies have suggested a role for miR-249 in promoting the development of lung cancer. For example, upregulated miR-429 expression was found to increase the sensitivity of epithelial ovarian cancer cells to cisplatin (DDP) treatment (13). Similarly, in another study, the overexpression of miR-429 induced DDP sensitivity in ovarian cancer cells (14). In fact, the chemoresistance of ovarian cancer organoids and cells has been found to be enhanced by controlling cellular senescence and glucose metabolism in ovarian cancer organoids and cells via the Aurora-A/SOX8/ forkhead box k1 (FOXK1) signaling axis (15). Furthermore, FOXK1 has also been identified to act as an oncogene in lung cancer (16).

The present study aimed to determine the role of circ-PVT1 in lung ADC and to investigate whether its effects in lung ADC were associated with the regulation of miR-429 and FOXK1. The current findings may provide novel insights into potential targets for the treatment of lung ADC.

\section{Materials and methods}

Cell culture and treatment. Human normal lung epithelial cells (BEAS-2B) and human lung ADC cells (HCC827, H1299, PC-9 and A549) were obtained from Shanghai Fuheng Biological Technology Co., Ltd. The DDP-resistant A549 cell line (A549/DDP) was obtained from JRDUN Biotechnology (Shanghai) Co., Ltd. All cells were cultured in DMEM (Gibco; Thermo Fisher Scientific, Inc.) supplemented with 10\% FBS (Gibco; Thermo Fisher Scientific, Inc.) and $1 \%$ penicillin-streptomycin (Thermo Fisher Scientific, Inc.). A549/DDP and A549 cells were cultured in medium supplemented with DDP (Sigma-Aldrich; Merck KGaA) at different concentrations $(0,1.25,2.5,5,10,20$ and $40 \mu \mathrm{M})$ at $37^{\circ} \mathrm{C}$ for $24 \mathrm{~h}$. Three cell-culture passages were studied and three replicates were performed in each passage.

Cell transfection. Short hairpin (sh)RNA-negative control (NC), shRNA-circ-PVT1\#1/2, overexpression (Oe)-NC, Oe-circ-PVT1, pcDNA3.1-NC and pcDNA3.1-FOXK1 were constructed by Shanghai GeneChem Co., Ltd. The NC used for the overexpression of FOXK1 was an empty pcDNA3.1 plasmid. The sequences for the miR-429 mimic, inhibitor and NC transfections were as follows: miR-429 mimic forward, 5'-UAAUACUGUCUGGUA AAACCGU-3' and reverse, 5'-GGUUUUACCAGACAGUAU UAUU-3'; NC mimic forward, 5'-UUCUCCGAACGUGUC ACGUUT-3' and reverse, 5'-ACGUGACACGUUCGGAGAATT -3'; miR-429 inhibitor, 5'-GCTGATTTAAAGGCTTAG-3'; and NC inhibitor, 5'-CAAATGTAGGTAGAGGA-3'.

miR-429 mimic, inhibitor and NC were obtained from Guangzhou RiboBio Biotechnology Co., Ltd. A549 cells $\left(3 \times 10^{5}\right)$ were seeded in 6-well plates and transfected with the aforementioned plasmids or oligonucleotides $(500 \mu \mathrm{M})$ using Lipofectamine $^{\circledR} 2000$ reagent (cat. no. 11668019; Invitrogen; Thermo Fisher Scientific, Inc.) according to the manufacturer's protocols. The time interval between transfection and subsequent experimentation was $48 \mathrm{~h}$.

Reverse transcription-quantitative PCR (RTqPCR). Total RNA was extracted from A549 cells using TRIzol ${ }^{\circledR}$ reagent (Invitrogen; Thermo Fisher Scientific, Inc.). Total RNA $(2 \mu \mathrm{g})$ was reverse-transcribed into cDNA using a Prime Script ${ }^{\mathrm{TM}}$ RT Master Mix reverse transcription kit (cat. no. RR036B; Takara Biotechnology Co., Ltd.) according to the manufacturer's protocol. qPCR was subsequently performed using TaqMan ${ }^{\mathrm{TM}}$ 2X Universal PCR Master mix without AmpErase ${ }^{\mathrm{TM}}$ UNG (Applied Biosystems; Thermo Fisher Scientific, Inc.) on an ABI 7500 Real-Time PCR system (Applied Biosystems; Thermo Fisher Scientific, Inc.). The following thermocycling conditions were used for qPCR: Initial denaturation at $95^{\circ} \mathrm{C}$ for $10 \mathrm{~min}$; followed by 40 cycles at $95^{\circ} \mathrm{C}$ for $15 \mathrm{sec}$ and $60^{\circ} \mathrm{C}$ for $1 \mathrm{~min}$. The following primers (Sangon Biotech Co., Ltd.) were used for qPCR: circ-PVT1 forward, 5'-ATCGGTGCCTCAGCGTTCGG-3' and reverse, 5'-CTGTCCTCGCCGTCACACCG-3'; miR-429 forward, 5'-ACACTCCAGCTGGGTAATACTGTCTGG TAA-3' and reverse, 5'-TGGTGTCGTGGAGTCG-3'; FOXK1 forward, 5'-ACGTTTGGTGACCAGAGGGA-3' and reverse, 5'-CGACAGAATTCAAGCCGCAC-3'; GAPDH forward, 5'-GAGTCAACGGATTTGGTCGTATTG-3' and reverse, 5'-CCTGGAAGATGGTGATGGGATT-3'; and U6 forward, 5'-AUAAAUCCCUUUACACCUCTT-3' and reverse, 5'-AAU AAAUCCCUUUACACCUCTT-3'. GAPDH and U6 were used as the internal controls for mRNA and miRNA, respectively. The relative mRNA expression levels were calculated using SDS version 2.0.1 software (Applied Biosystems; Thermo Fisher Scientific, Inc.). Quantification of the relative mRNA expression levels were performed using the $2^{-\Delta \Delta \mathrm{Cq}}$ method (17).

Western blotting. Total protein was extracted from A549 cells using RIPA lysis buffer (Beyotime Institute of Biotechnology) supplemented with protease and phosphatase inhibitors. Protein was quantified using the BCA method and $30 \mu \mathrm{g}$ protein/lane was loaded and separated via 12\% SDS-PAGE. The separated proteins were subsequently transferred onto nitrocellulose membranes and blocked with 5\% non-fat milk at room temperature for $1 \mathrm{~h}$. The membranes were then incubated with primary antibodies against matrix metalloproteinase (MMP)2 (cat. no. ab92536; 1:1,000; Abcam), MMP9 (cat. no. ab76003; 1:1,000; Abcam), FOXK1 (cat. no. ab18196; 1:1,000; Abcam) and GAPDH (cat.no. ab9485; 1:2,500; Abcam) at $4^{\circ} \mathrm{C}$ overnight. Following the primary antibody incubation, the membranes were washed with TBS with Tween-20 $(0.05 \%)$ twice and incubated with HRP-conjugated goat anti-rabbit IgG (cat. no. ab6721; 1:2,000; Abcam) secondary antibody at room temperature for $1 \mathrm{~h}$. Protein bands were visualized using Immobilon $^{\mathrm{TM}}$ Western Chemiluminescent HRP substrate (EMD Millipore). ImageJ software (version 1.8.0; National Institutes of Health) was used for densitometric analysis.

Transwell assay. The 96-well Transwell chambers were precoated with Matrigel (BD Biosciences) overnight at $37^{\circ} \mathrm{C}$. A549 cells $\left(0.5 \times 10^{6}\right.$ cells $\left./ \mathrm{ml}\right)$ were suspended in serum-free 
DMEM and plated into the upper chamber. The lower chamber of the 96-well Transwell plate (cat. no. 3380; Corning, Inc.) was filled with DMEM supplemented with 10\% FBS (18). Following $24 \mathrm{~h}$ of incubation at $37^{\circ} \mathrm{C}$, the invasive cells were fixed with $4 \%$ paraformaldehyde (Sigma-Aldrich; Merck $\mathrm{KGaA}$ ) for $10 \mathrm{~min}$ at room temperature and stained with $0.1 \%$ crystal violet (Sigma-Aldrich; Merck KGaA) for $15 \mathrm{~min}$ at room temperature. Stained cells were visualized using an inverted microscope (Olympus Corporation) in five randomly selected fields of view (magnification, x100).

Wound healing assay. A549 cells ( $3 \times 10^{4}$ cells/well) were plated into a 96-well plate and cultured until they reached $100 \%$ confluency. A $200-\mu 1$ sterile pipette tip was subsequently used to make a single vertical scratch in the cell monolayer. The cells were then washed with PBS three times to remove non-adherent cells and cultured in serum-free DMEM at $37^{\circ} \mathrm{C}$ for $24 \mathrm{~h}$. Cells were visualized and imaged under a light microscope (magnification, x100).

Colony formation assay. A549 cells ( $2 \times 10^{3}$ cells/well) were seeded into 96-well plates and cultured in a humidified incubator at room temperature. Following 2 weeks of incubation, the cells were fixed with $4 \%$ paraformaldehyde for $30 \mathrm{~min}$ at room temperature and stained with $0.5 \%$ gentian violet for $1 \mathrm{~h}$ at room temperature. Stained cells were observed and imaged with a camera (Canon, Inc.).

MTT assay. A549 cells ( $3 \times 10^{3}$ cells/well) were seeded into a 96-well culture plate containing DMEM supplemented with $10 \%$ FBS and exposed to different doses of DDP $(1.25,2.5$, 5, 10, 20 and $40 \mu \mathrm{M}$ ) for $48 \mathrm{~h}$. Following incubation, $10 \mu \mathrm{l}$ MTT $(10 \mathrm{mg} / \mathrm{ml})$ was added/well and incubated for $4 \mathrm{~h}$. The culture medium was subsequently removed, and $100 \mu \mathrm{l}$ DMSO (Beyotime Institute of Biotechnology) was added to the wells to dissolve the purple formazan crystals. Following incubation for $30 \mathrm{~min}$, the optical density (OD) of each well was measured using a microplate reader at a wavelength of $570 \mathrm{~nm}$.

Caspase-3 activity assay. Caspase-3 activity was measured in A549 cells using a colorimetric assay kit (cat. no. C1115; Beyotime Institute of Technology), according to the manufacturer's protocol.

Dual-luciferase reporter assay. The binding sites between miR-429 and circ-PVT1, and between FOXK1 and miR-429 were predicted using ENCORI (http://starbase. sysu.edu.cn/index.php). The wild-type (WT) or mutant (MUT) 3'-untranslated regions (UTRs) of circ-PVT1 and FOXK1 were constructed and cloned downstream of the pmirGLO firefly luciferase reporter vector (Promega Corporation), which were co-transfected with miR-429 mimic or mimic-NC into A549 cells using Lipofectamine 2000 reagent. Mutations in the 3'-UTRs were generated using a QuikChange Multi Site-Directed Mutagenesis kit (Agilent Technologies, Inc.). The relative luciferase activity was measured using a Dual Luciferase Reporter Assay kit (Promega Corporation) at $48 \mathrm{~h}$ post-transfection. Firefly luciferase activity was normalized to Renilla luciferase activity.
Statistical analysis. Statistical analysis was performed using SPSS 22.0 software (IBM Corp.) and GraphPad Prism 7 software (GraphPad Software, Inc.). All experiments were performed in triplicate and data are expressed as the mean \pm SD. Statistical differences between two groups were determined using an unpaired student's t-test, whereas statistical differences among multiple groups were performed using one-way ANOVA followed by Tukey's post hoc test. The correlation between miR-429 expression and circ-PVT1 expression in human normal lung epithelial cells and human lung ADC cells was determined using Pearson's correlation analysis. $\mathrm{P}<0.05$ was considered to indicate a statistically significant difference.

\section{Results}

Knockdown of circ-PVT1 inhibits the proliferation, invasion and migration of A549 cells. To validate the role of circ-PVT1 in the progression of lung ADC, the mRNA expression levels of circ-PVT1 in human normal lung epithelial cells and lung ADC cells were analyzed using RT-qPCR. The expression levels of circ-PVT1 were significantly upregulated in lung ADC cells compared with human normal lung epithelial cells (Fig. 1A). The expression levels of circ-PVT1 were the highest in A549 cells; therefore, A549 cells were selected for use in subsequent experiments. The expression of circ-PVT1 in A549 cells was knocked down by transfection with shRNA-circ-PVT1. The expression levels of circ-PVT1 were downregulated to a greater extent in cells transfected with shRNA-circ-PVT1\#2 compared with shRNA-circ-PVT1\#1; therefore, shRNA-circ-PVT1\#2 was used for the following transfections (Fig. 1B). Compared with the control and shRNA-NC groups, cell viability (Fig. 1C), proliferation (Fig. 1D and E), invasion and migration (Fig. 2A-D) were all decreased in the shRNA-circ-PVT1 group. Moreover, the expression levels of MMP2 and MMP9 were significantly downregulated in A549 cells transfected with shRNA-circ-PVT1 (Fig. 2E and F). These results suggested that the knockdown of circ-PVT1 may inhibit the proliferation, invasion and migration of A549 cells.

\section{Expression levels of circ-PVT1 are upregulated in A549/DDP} cells and are involved in DDP-induced apoptosis. The association between circ-PVT1 and DDP resistance was investigated in A549 and A549/DDP cells. The expression levels of circ-PVT1 were upregulated in A549/DDP cells compared with A549 cells, suggesting the involvement of circ-PVT1 in DDP resistance (Fig. 3A). To determine the effects of circ-PVT1 on DDP-resistant lung ADC cells, A549/DDP cells were transfected with shRNA-circ-PVT1 and A549 cells were transfected with Oe-circ-PVT1 plasmids (Fig. 3B and E). The cells were then treated with different concentrations of DDP. The results of the MTT assay revealed that the transfection of cells with shRNA-circ-PVT1 significantly reduced the viability of A549/DDP cells treated with increasing doses of DDP compared with control and shRNA-NC-transfected cells (Fig. 3C). In addition, the transfection of cells with the Oe-circ-PVT1 plasmid increased the viability of A549 cells treated with increasing doses of DDP (Fig. 3F). Notably, treatment with 5 and $10 \mu \mathrm{M}$ DDP markedly affected cell viability at the early stage. Caspase-3 activity was also increased following DDP treatment in 

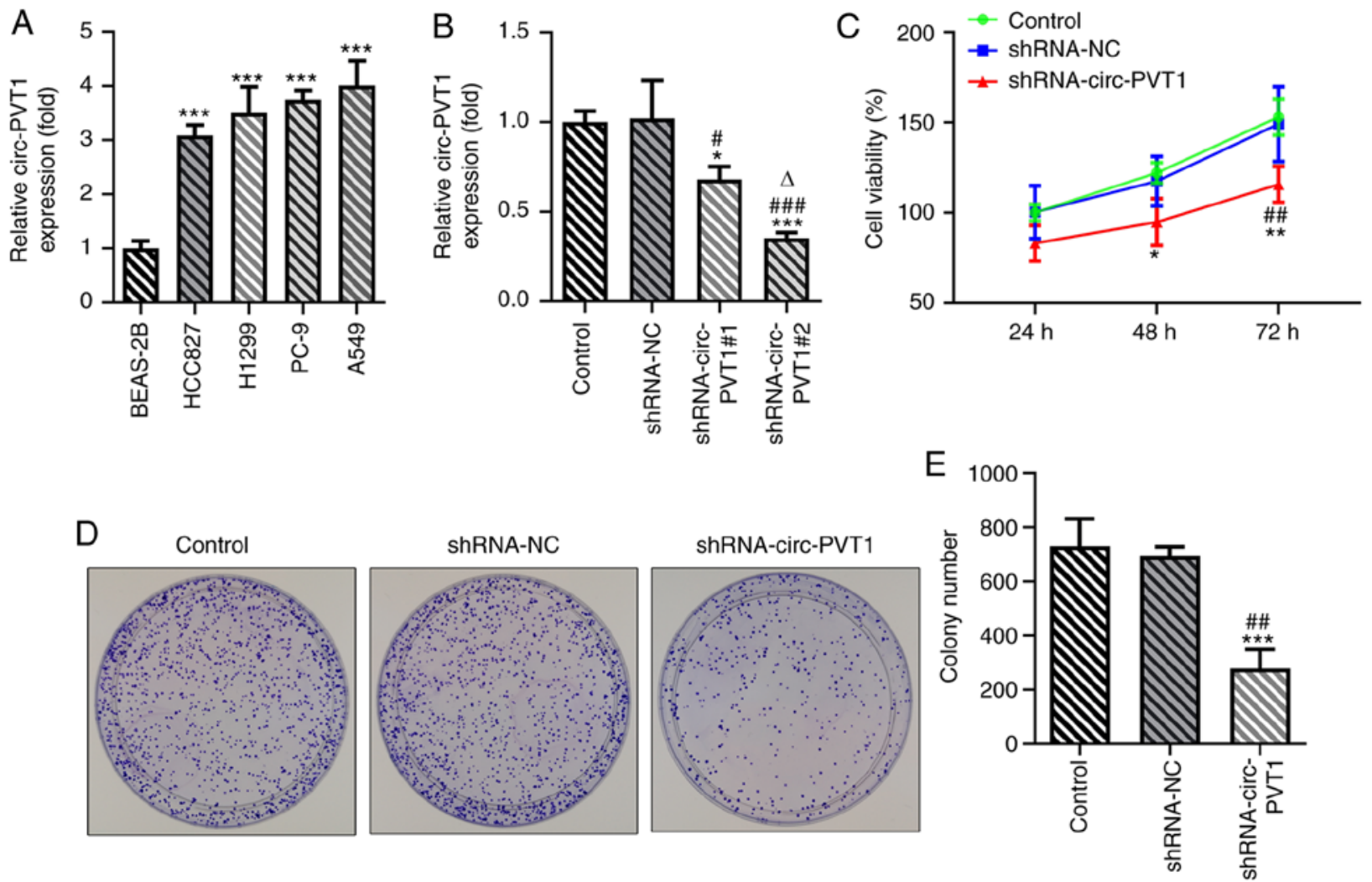

Figure 1. Knockdown of circ-PVT1 inhibits the proliferation of A549 cells. (A) The mRNA levels of circ-PVT1 in human normal lung epithelial cells (BEAS-2B) and human lung ADC cells (HCC827, H1299, PC-9, A549) were measured via reverse transcription-quantitative PCR. ${ }^{* * *} \mathrm{P}<0.001$ vs. BEAS-2B group. (B) After knockdown of circ-PVT1, the expression of circ-PVT1 was detected. ${ }^{*} \mathrm{P}<0.05$ and ${ }^{* * *} \mathrm{P}<0.001$ vs. Control group; ${ }^{*} \mathrm{P}<0.05$ and ${ }^{\# \# \#} \mathrm{P}<0.001$ vs. shRNA-NC group; ${ }^{\triangle} \mathrm{P}<0.05$ vs. shRNA-circ-PVT1\#1 group. (C) Cell viability was measured via a Cell Counting Kit-8 assay after knockdown of circ-PVT1. (D and E) The colony formation ability of lung ADC cells was determined. ${ }^{*} \mathrm{P}<0.05,{ }^{* *} \mathrm{P}<0.01$ and ${ }^{* * * *} \mathrm{P}<0.001$ vs. Control group; ${ }^{\# \#} \mathrm{P}<0.01$ vs. shRNA-NC group. circ-PVT1, circular RNA plasmacytoma variant translocation 1; ADC, adenocarcinoma; shRNA, short hairpin RNA; NC, negative control.

A549/DDP cells transfected with shRNA-circ-PVT1 (Fig. 3D), while caspase-3 activity was decreased following DDP treatment in A549 cells transfected with the Oe-circ-PVT1 plasmid (Fig. 3G). These findings indicated that the expression levels of circ-PVT1 may be upregulated in A549/DDP cells and involved in DDP-induced apoptosis.

circ-PVT1 negatively regulates the expression levels of $m i R-429$. The binding sites between miR-429 and circ-PVT1 are shown in Fig. 4A. The relative luciferase activity was reduced in the miR-429 mimic + circ-PVT1 WT group compared with the mimic-NC + circ-PVT1 WT group, while it remained unchanged between the miR-429 mimic + circ-PVT1 MUT group and mimic-NC + circ-PVT1 groups (Fig. 4B). Notably, miR-429 expression was decreased in lung ADC cells compared with human normal lung epithelial cells (Fig. 4C). miR-429 expression was negatively correlated with circ-PVT1 expression in human normal lung epithelial cells and human lung ADC cells using a Pearson's correlation analysis (Fig. 4D). The expression levels of miR-429 were significantly upregulated in cells transfected with the shRNA-circ-PVT1 compared with the control groups (Fig. 4E). The expression levels of miR-429 were upregulated in the miR-429 mimic group compared with the mimic-NC group, while they were decreased in the miR-429 inhibitor group compared with the $\mathrm{NC}$ inhibitor group (Fig. 4F). These results suggested that circ-PVT1 may negatively regulate the expression of miR-429.
FOXK1 is a target gene of miR-429. The binding sites between FOXK1 and miR-429 were predicted and are presented in Fig. 5A. The results of the dual-luciferase reporter assay validated the hypothesis that miR-429 may regulate the expression of FOXK1 (Fig. 5B). The expression levels of FOXK1 were subsequently determined using RT-qPCR and western blotting in BEAS-2B cells and multiple lung ADC cell lines. The expression levels of FOXK1 were upregulated in lung ADC cells, and the expression levels of FOXK1 were the highest in A549 cells (Fig. 5C and D). As shown in Fig. 5E and F, transfection with the miR-429 mimic downregulated the expression levels of FOXK1 in A549 cells. Following the knockdown of circ-PVT1 in A549 cells, FOXK1 expression levels were also downregulated (Fig. 5G and H).

circ-PVT1/miR-429/FOXK1 signaling axis promotes the proliferation, invasion and migration of A549 cells, which may occur through DDP-induced apoptosis. Finally, whether circ-PVT1 could regulate the expression of FOXK1 via sponging miR-429 to affect the cellular behaviors of A549 cells was investigated. The pcDNA3.1-FOXK1 vector was constructed and transfected into cells; the expression levels of FOXK1 were upregulated in cells transfected with the pcDNA3.1-FOXK1 vector compared with the control or empty vector groups (Fig. 6A and B). Transfection of cells with shRNA-circ-PVT1 decreased the cell viability, proliferation, invasion and migration, which was subsequently reversed by transfection with either the 
A
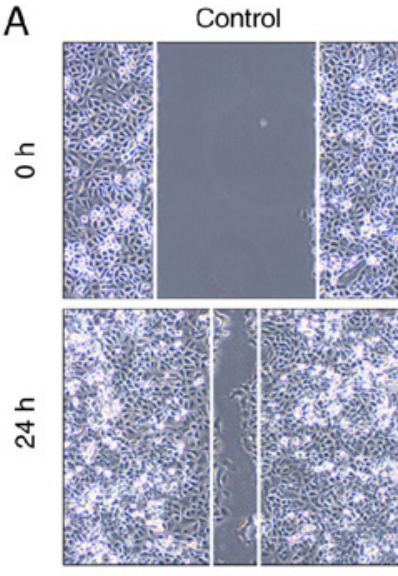

C
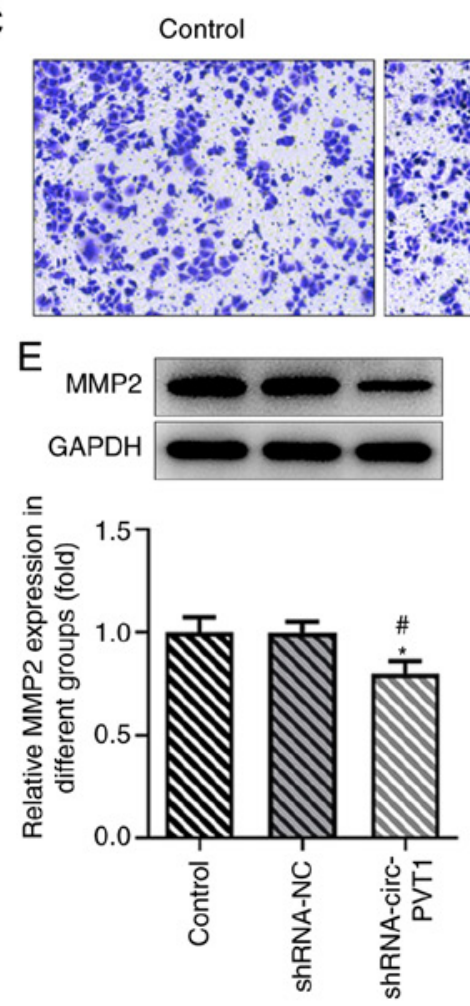

ShRNA-NC
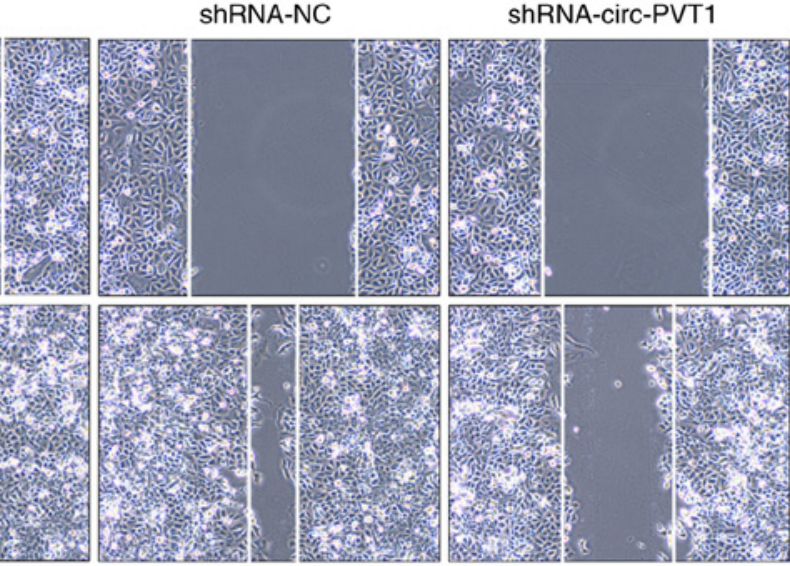

ShRNA-NC
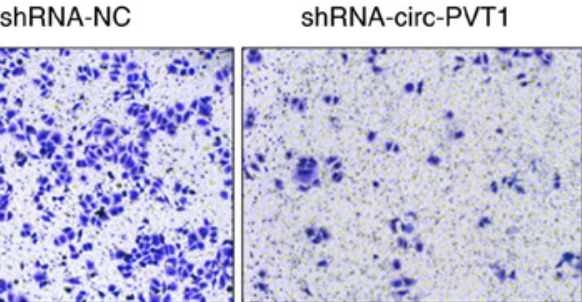
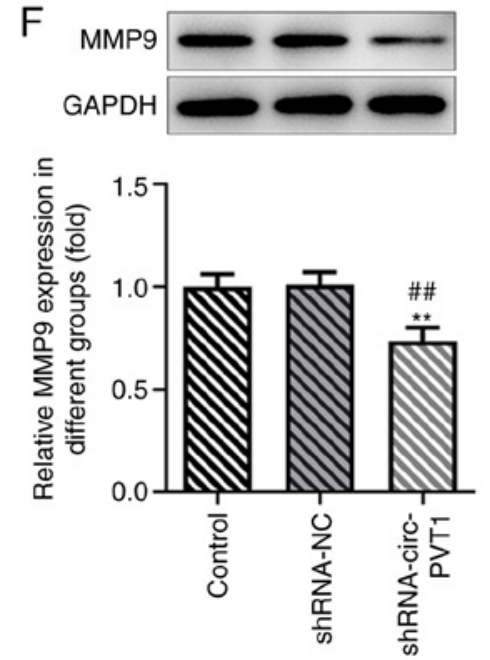
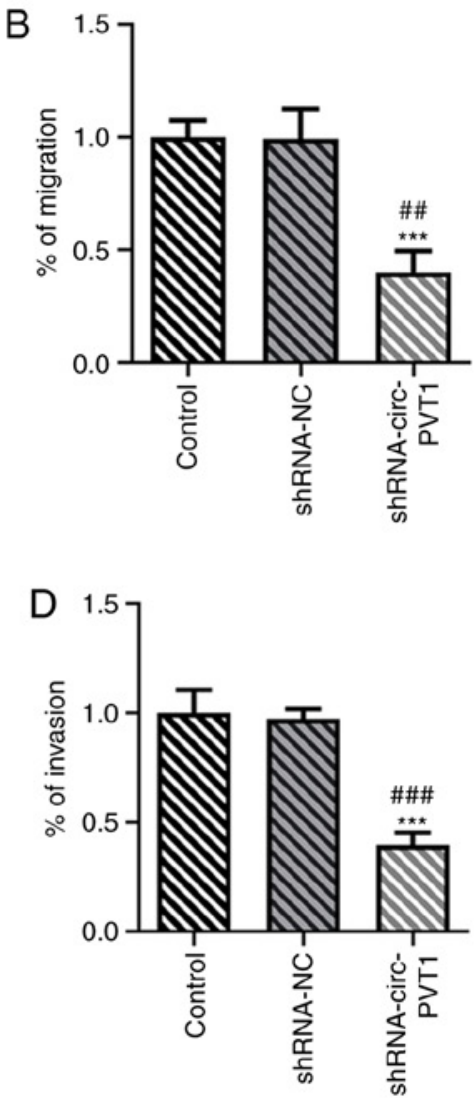

Figure 2. Knockdown of circ-PVT1 inhibits the invasion and migration of A549 cells. (A-D) The invasion and migration of A549 cells were detected after knockdown of circ-PVT1. (E and F) Western blotting was performed to measure the mRNA expression levels of MMP2 and MMP9. *P<0.05, ${ }^{* *} \mathrm{P}<0.01$ and ${ }^{* * *} \mathrm{P}<0.001$ vs. Control group; ${ }^{\#} \mathrm{P}<0.05,{ }^{\# \#} \mathrm{P}<0.01$ and ${ }^{\# \# \#} \mathrm{P}<0.001$ vs. shRNA-NC group. circ-PVT1, circular RNA plasmacytoma variant translocation 1 ; shRNA, short hairpin RNA; NC, negative control; MMP, matrix metalloproteinase.

miR-429 inhibitor or pcDNA3.1-FOXK1 plasmid (Fig. 6C-I). The expression levels of MMP2 and MMP9 were also downregulated by shRNA-circ-PVT1, while transfection with the miR-429 inhibitor or pcDNA3.1-FOXK1 could upregulate the expression levels (Fig. 6J and $\mathrm{K}$ ). In addition, the shRNA-circ-PVT1-induced reduction in cell viability and increased caspase-3 activity in A549/DDP cells were both partially abolished by transfection with the miR-429 inhibitor (Fig. 7A and B). The Oe-circ-PVT1-induced increase in cell viability and decreased caspase-3 activity in A549 cells were also partially abolished by transfection with the miR-429 inhibitor or pcDNA3.1-FOXK1 (Fig. 7C and D). These results suggested that the circ-PVT1/miR-429/FOXK1 signaling axis may promote the proliferation, invasion and migration of A549 cells, which may occur through DDP-induced apoptosis.

\section{Discussion}

circRNAs are ncRNAs that have high stability and tissue-specific expression patterns (19). However, to the best of our knowledge, the functions of the majority of circRNAs are poorly understood. Increasingly mature high-throughput sequencing technologies have provided evidence to suggest that circRNAs may be associated with various diseases, including cancer (20). To date, circRNAs have been reported to participate in the development of lung cancer. For example, circRNA itchy E3 ubiquitin protein ligase was observed to inhibit the proliferation of lung cancer via inactivating Wnt/ $\beta$-catenin signaling (21). circ-homeodomain interacting protein kinase 3 , as an oncogene, has been found to modulate autophagy and has been suggested to be a potential prognostic 

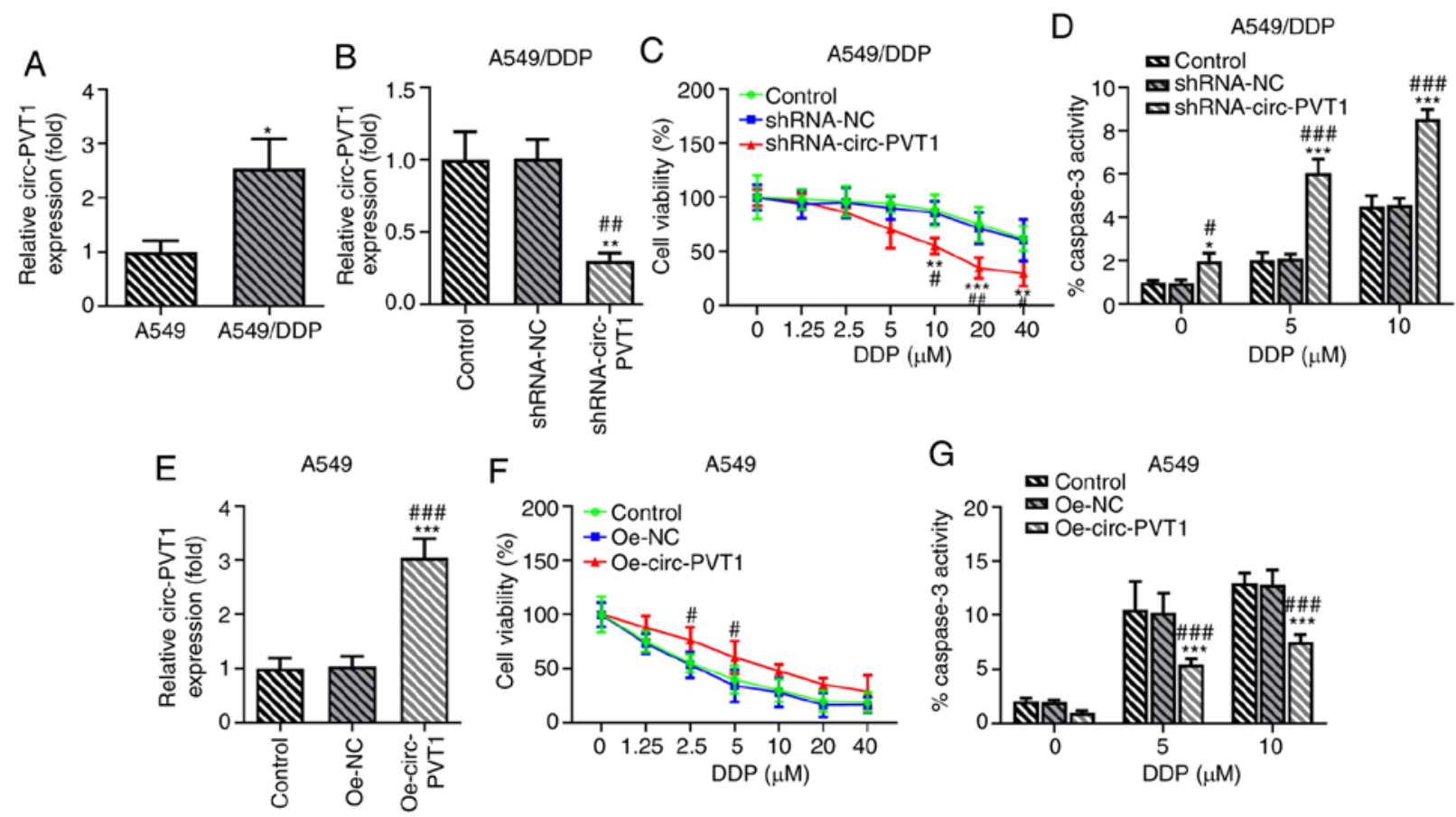

Figure 3. circ-PVT1 is upregulated in A549/DDP cells and is involved in DDP-induced apoptosis. (A) The mRNA levels of circ-PVT1 in A549 and A549/DDP cells were measured via reverse transcription-quantitative PCR. " $\mathrm{P}<0.05$ vs. A549 group. (B) The expression of circ-PVT1 in A549/DDP cells after knockdown of circ-PVT1. ${ }^{* *} \mathrm{P}<0.01$ vs. Control group; ${ }^{\# \#} \mathrm{P}<0.01$ vs. shRNA-NC group. (C) The cell viability of A549/DDP cells after treatment with different concentrations of DDP was measured. ${ }^{* *} \mathrm{P}<0.01$ and ${ }^{* * * *} \mathrm{P}<0.001$ vs. Control group; ${ }^{\#} \mathrm{P}<0.05$ and ${ }^{\# \#} \mathrm{P}<0.01$ vs. shRNA-NC group. (D) The activity of caspase-3 was measured after knockdown of circ-PVT1 in A549/DDP cells. " $\mathrm{P}<0.05$ and ${ }^{* * * *} \mathrm{P}<0.001$ vs. Control group; ${ }^{*} \mathrm{P}<0.05$ and ${ }^{\# \# \#} \mathrm{P}<0.001$ vs. shRNA-NC group. (E) The expression of circ-PVT1 was detected after circ-PVT1 was overexpressed in A549 cells. ${ }^{* * * *} \mathrm{P}<0.001$ vs. Control group; ${ }^{\# \# \# ~} \mathrm{P}<0.001$ vs. Oe-NC group. (F) The cell viability of A549 cells after treatment with different concentrations of DDP was measured. ${ }^{~} \mathrm{P}<0.05$ vs. Oe-NC group. (G) The activity of caspase-3 was measured after circ-PVT1 was overexpressed in A549 cells. ${ }^{* * *} \mathrm{P}<0.001$ vs. Control group; ${ }^{\# \# /} \mathrm{P}<0.001$ vs. Oe-NC group. circ-PVT1, circular RNA plasmacytoma variant translocation 1; DDP, cisplatin; shRNA, short hairpin RNA; NC, negative control; Oe, overexpression.

A circ-PVT1: $5^{\prime}$ cCccaUGUACU---CAGUAUUa $3^{\prime}$
hsa-miR-429: $3^{\prime}$ UgccaAII: 11111111

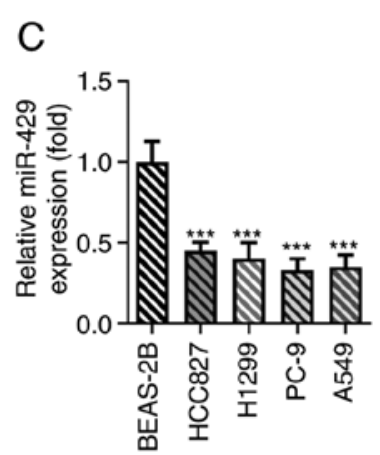

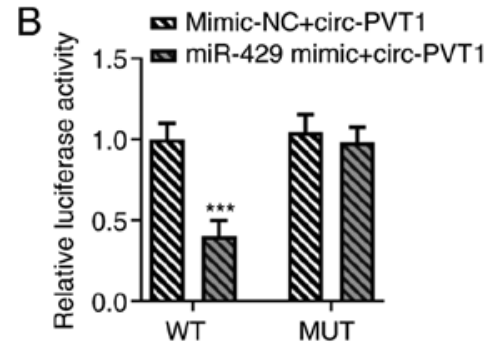

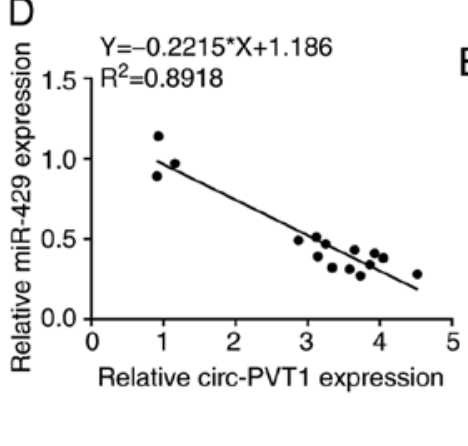

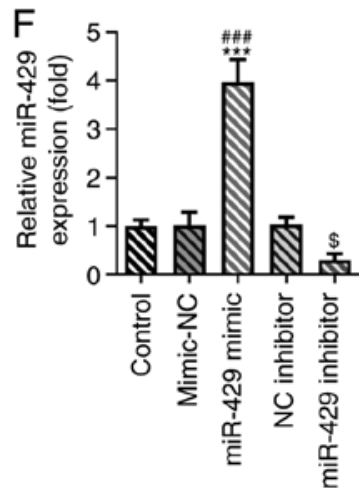

Figure 4. circ-PVT1 negatively regulates the expression of miR-429. (A) It was predicted that miR-429 may target the 3'UTR of circ-PVT1. (B) The relationship between circ-PVT1 and miR-429 was measured via a dual-luciferase reporter assay. ${ }^{* * * *} \mathrm{P}<0.001$ vs. miR-429 mimic + circ-PVT1 MUT group. (C) The mRNA levels of miR-429 in human normal lung epithelial cells (BEAS-2B) and human lung adenocarcinoma cells (HCC827, H1299, PC-9, A549) were measured via reverse transcription-quantitative PCR. ${ }^{* * *} \mathrm{P}<0.001$ vs. BEAS-2B group. (D) The correlation between circ-PVT1 and miR-429 was determined using Pearson's correlation coefficient in human normal lung epithelial cells and human lung ADC cells. (E) The mRNA expression of miR-429 after knockdown of circ-PVT1 was determined. ${ }^{* * *} \mathrm{P}<0.001$ vs. Control group; ${ }^{\# \# \#} \mathrm{P}<0.001$ vs. shRNA-NC group. (F) Proof of transfection for miR- 429 mimic and inhibitor transfections. ${ }^{* * *} \mathrm{P}<0.001$ vs. Control group; ${ }^{\# \# "} \mathrm{P}<0.001$ vs. mimic-NC group; ${ }^{\mathrm{S}} \mathrm{P}<0.05$ vs. $\mathrm{NC}$ inhibitor group. circ-PVT1, circular RNA plasmacytoma variant translocation 1 ; miR, microRNA; shRNA, short hairpin RNA; NC, negative control; Oe, overexpression; WT, wild-type; MUT, mutant. 
A $\begin{array}{cc}\text { FOXK1: } 5^{\prime} \text { uuucaagugggAUACAGUAUUu } & 3^{\prime} \\ & \end{array}$
B

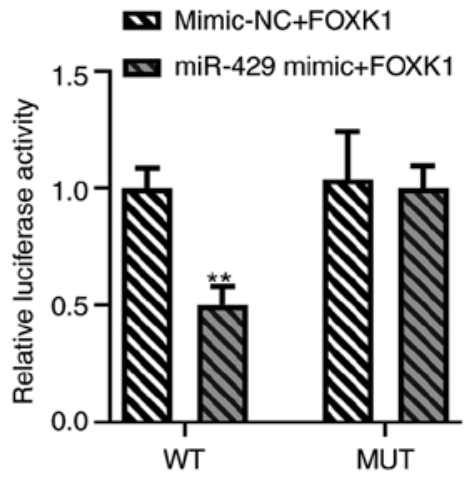

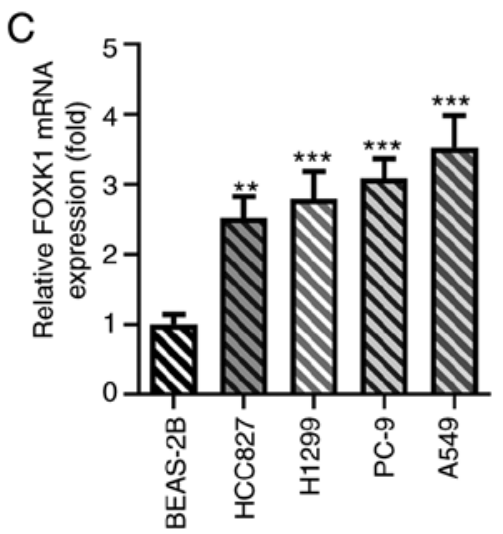
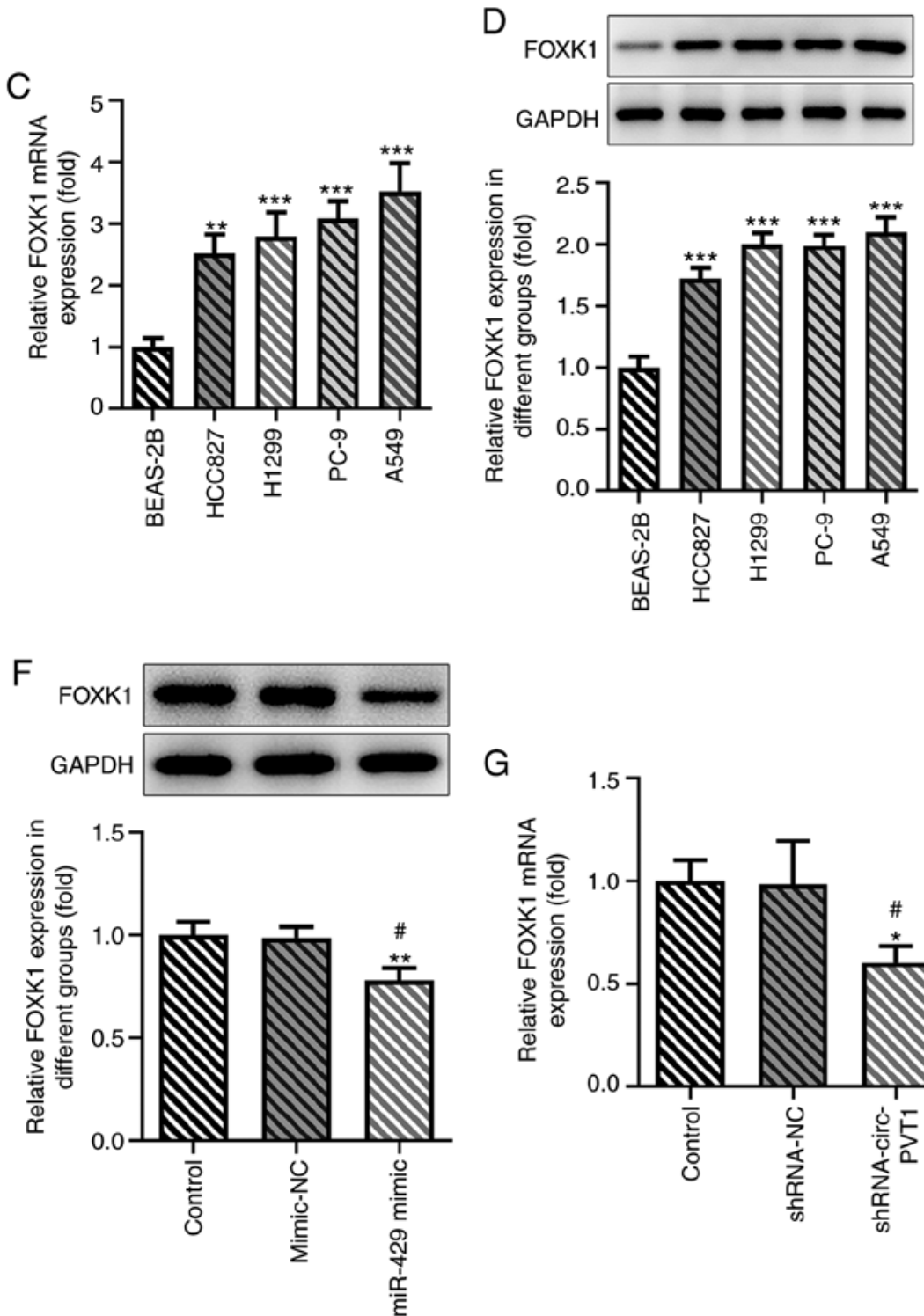

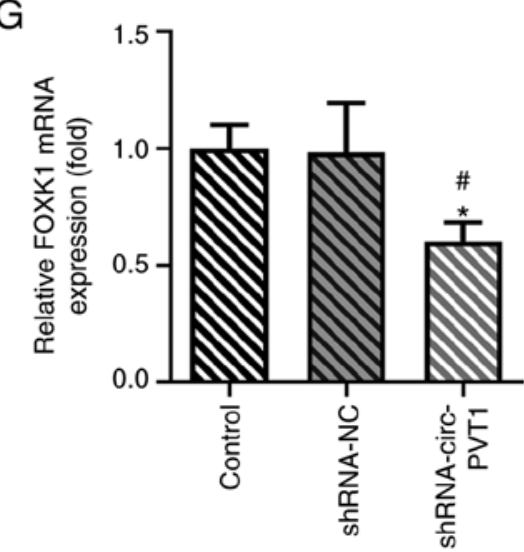

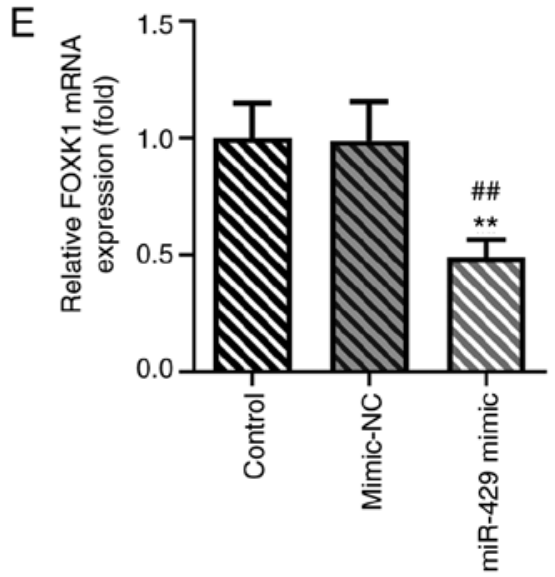
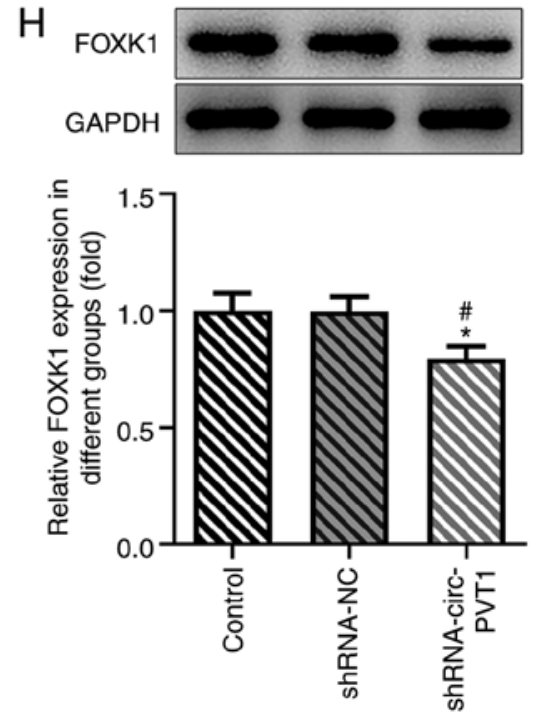

Figure 5. FOXK1 is a target of miR-429. The putative binding sites between FOXK1 and hsa-miR-429 were (A) predicted and (B) confirmed via a dual-luciferase reporter assay. ${ }^{* *} \mathrm{P}<0.01$ vs. mimic-NC + FOXK1 group. (C and D) The expression of FOXK1 in human normal BEAS-2B and human lung adenocarcinoma cells was measured via RT-qPCR and western blotting. ${ }^{* *} \mathrm{P}<0.01$ and ${ }^{* * * *} \mathrm{P}<0.001$ vs. BEAS-2B group. (E and F) The expression of FOXK1 after miR-429 overexpression in A549 cells was measured via RT-qPCR and western blotting. ${ }^{* *} \mathrm{P}<0.01$ vs. Control group; ${ }^{\#} \mathrm{P}<0.05$ and ${ }^{\# \#} \mathrm{P}<0.01$ vs. mimic-NC group. (G and $\left.\mathrm{H}\right)$ The expression of FOXK1 after knockdown of circ-PVT1 expression in A549 cells was measured via RT-qPCR and western blotting. "P<0.05 vs. Control group; "P<0.05 vs. shRNA-NC group. FOXK1, forkhead box k1; miR, microRNA; NC, negative control; RT-qPCR, reverse transcription-quantitative PCR; circ-PVT1, circular RNA plasmacytoma variant translocation 1; shRNA, short hairpin RNA; WT, wild-type; MUT, mutant.

marker in lung cancer (22). In addition, circ-mannosidase $\alpha$ class $2 \mathrm{~B}$ member 2 has been reported to sponge miR-1275 and promote the development of lung cancer (23). To date, the role of circ-PVT1 has been extensively studied in multiple types of 


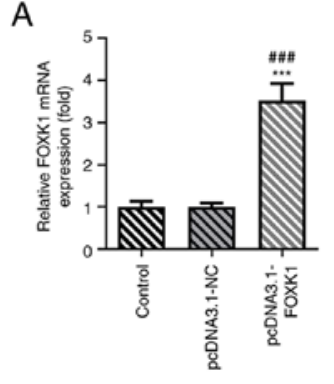

B

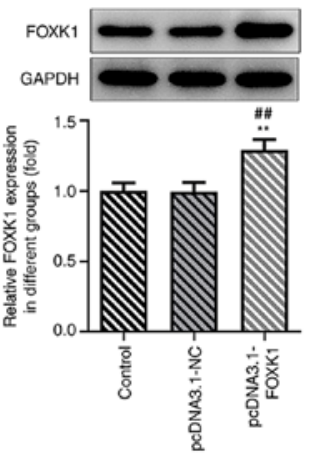

C $\rightarrow$ - ShRNA.NC

-- shRNA-circ-PVT1

\# shRNA-Circ-PVT1+NC inhibitor

- shPNA-Circ-PVT $1+\mathrm{NC}$ inhibitor

$\rightarrow \rightarrow$ shRNA-Circ-PVT 1+miR-429 inhibitor

$\left.{ }^{200}\right] \rightarrow$ - shRNA-circ-PVT 1+pCDNA3.1-FOXK1

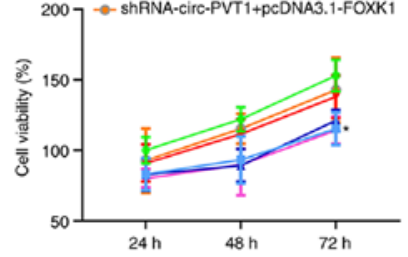

D

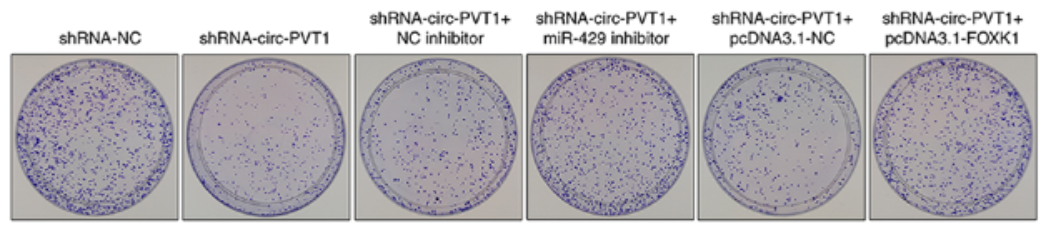

E

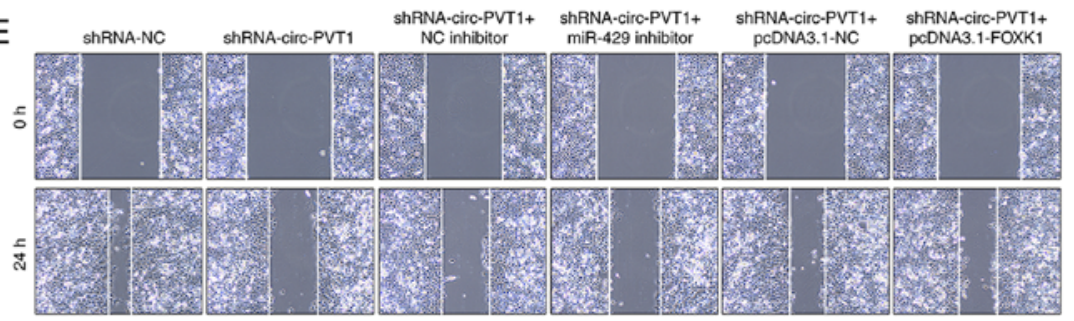

$\mathrm{F}$

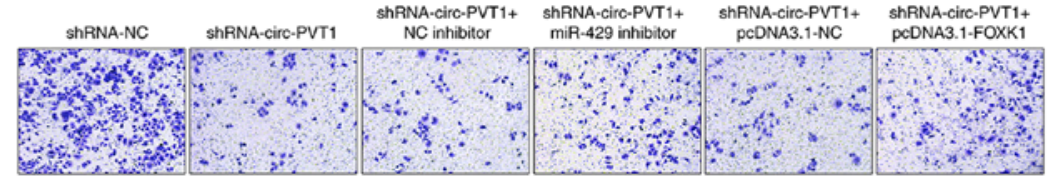

G

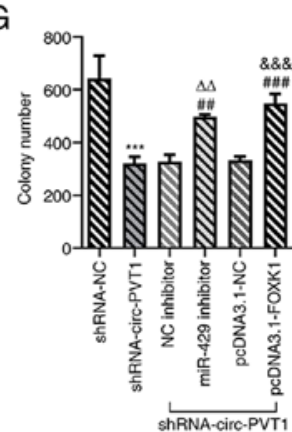

$\mathrm{H}$
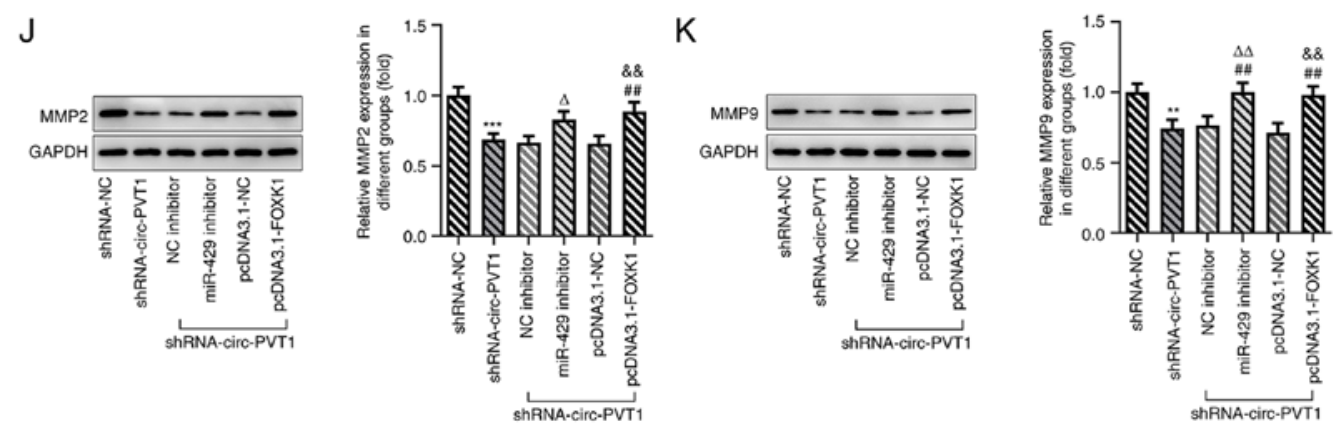

Figure 6. circ-PVT1/miR-429/FOXK1 axis promotes the proliferation, invasion and migration of A549 cells and involves cisplatin-induced apoptosis. (A and B) The expression of FOXK1 following transfection with pcDNA3.1-FOXK1 was determined via reverse transcription-quantitative PCR and western blotting. ${ }^{* *} \mathrm{P}<0.01$ and ${ }^{* * *} \mathrm{P}<0.001$ vs. Control group; ${ }^{\# \#} \mathrm{P}<0.01$ and ${ }^{\# \#} \mathrm{P}<0.001$ vs. pcDNA3.1-NC group. (C) The cell viability of A549 cells was measured after the cells were transfected with the corresponding plasmids. " $\mathrm{P}<0.05$ vs. shRNA-NC group. (D-I) The proliferation, migration and invasion of A549 cells were detected by colony formation, wound healing and Transwell assays, respectively. ${ }^{* * *} \mathrm{P}<0.001$ vs. shRNA-NC group; ${ }^{\# \#} \mathrm{P}<0.01$ and ${ }^{\# \# \#} \mathrm{P}<0.001$ vs. shRNA-circ-PVT1 group; ${ }^{\Delta \Delta} \mathrm{P}<0.01$ vs . shRNA-circ-PVT1 + NC inhibitor group; \&\&\& $\mathrm{P}<0.001 \mathrm{vs.} \mathrm{shRNA-circ-PVT1} \mathrm{+} \mathrm{pcDNA3.1-NC} \mathrm{group.} \mathrm{(J} \mathrm{and} \mathrm{K)} \mathrm{The} \mathrm{expression} \mathrm{levels} \mathrm{of} \mathrm{MMP2} \mathrm{and} \mathrm{MMP9} \mathrm{were}$ measured after the cells were transfected with the corresponding plasmids. ${ }^{* *} \mathrm{P}<0.01$ and ${ }^{* * * *} \mathrm{P}<0.001$ vs. shRNA-NC group; ${ }^{\# \#} \mathrm{P}<0.01$ vs. shRNA-circ-PVT1 group; ${ }^{\Delta} \mathrm{P}<0.05$ and ${ }^{\Delta} \mathrm{P}<0.01$ vs. shRNA-circ-PVT1 + NC inhibitor group; \& $\mathrm{P}<0.01$ vs. shRNA-circ-PVT1 + pcDNA3.1-NC group. FOXK1, forkhead box k1; miR, microRNA; NC, negative control; circ-PVT1, circular RNA plasmacytoma variant translocation 1; shRNA, short hairpin RNA; MMP, matrix metalloproteinase. 
A

A549/DDP

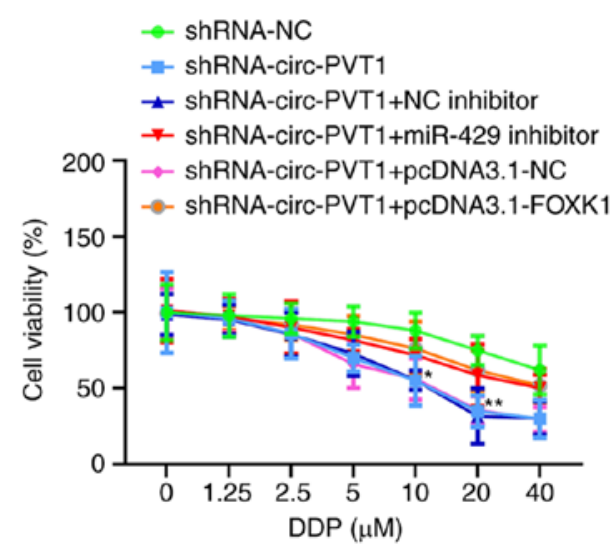

C

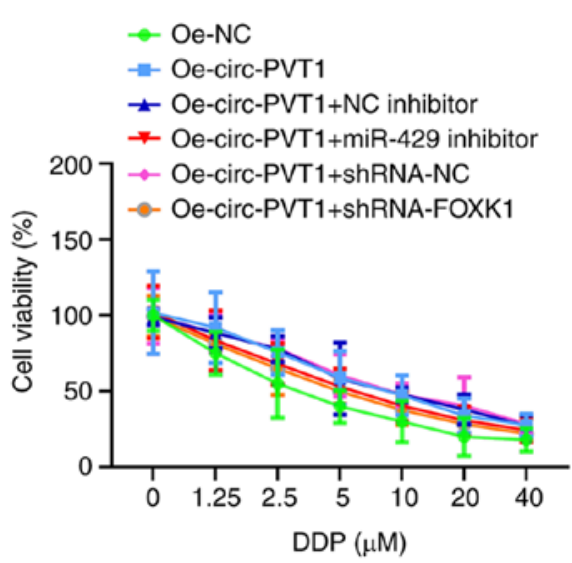

B
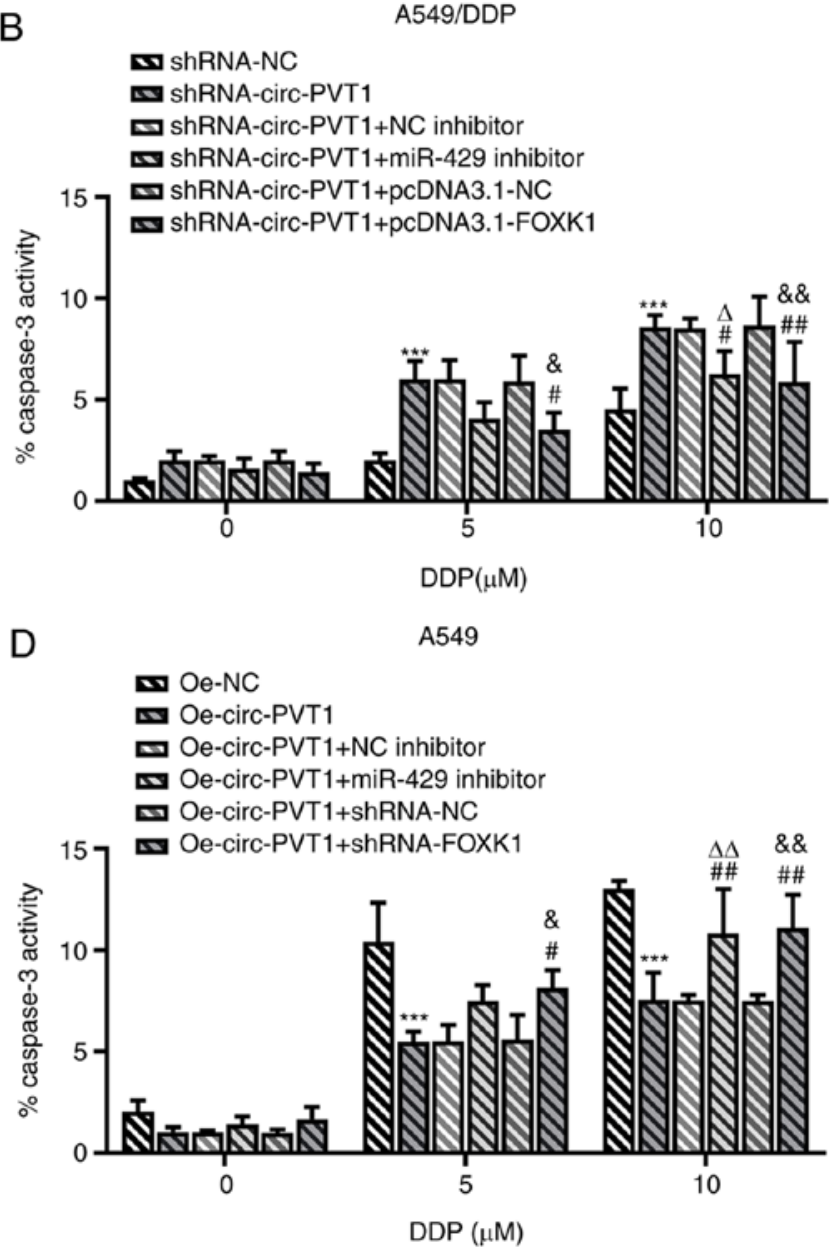

Figure 7. circ-PVT1/miR-429/FOXK1 axis is involved in DDP-induced apoptosis. (A) The cell viability and (B) activity of caspase-3 were detected after A549/DDP cells were transfected with the corresponding plasmids. ${ }^{*} \mathrm{P}<0.05,{ }^{* *} \mathrm{P}<0.01$ and ${ }^{* * *} \mathrm{P}<0.001$ vs. shRNA-NC group; ${ }^{*} \mathrm{P}<0.05$ amd

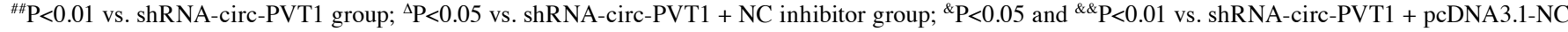
group. (C) The cell viability and (D) activity of caspase-3 were detected after A549 cells were transfected with the corresponding plasmids. ${ }^{* * *} \mathrm{P}<0.001 \mathrm{vs}$. Oe-NC group; ${ }^{\# \#} \mathrm{P}<0.01$ vs. Oe-circ-PVT1 group; ${ }^{\Delta \Lambda} \mathrm{P}<0.01$ vs. Oe-circ-PVT1 $+\mathrm{NC}$ inhibitor group; ${ }^{\circledR} \mathrm{P}<0.05$ and ${ }^{\&} \mathrm{P}<0.01$ vs. Oe-circ-PVT1 + shRNA-NC group FOXK1, forkhead box k1; miR, microRNA; NC, negative control; circ-PVT1, circular RNA plasmacytoma variant translocation 1; DDP, cisplatin; shRNA, short hairpin RNA; Oe, overexpression.

cancer; however, to the best of our knowledge, its role in lung cancer has not been discussed.

The aberrant expression of miRNAs has been reported to be involved in multiple processes of cancer cells, including proliferation, apoptosis and differentiation (24). Accumulating evidence has demonstrated the significant role of miRNAs in the progression of NSCLC. For example, the miR-200 family was shown to be implicated in lung cancer development and drug sensitivity (24). Emerging evidence has also supported the inhibitory role of miR-429, a member of the miR-200 family, on the expression of tumor suppressor genes, such as PTEN and TIMP metallopeptidase inhibitor 2, to enhance the proliferation, migration and invasion of cells (25). Another study also reported a significant role for miR-429 in promoting the proliferation of NSCLC cells (25). Thus, the role of miR-429 in lung ADC cells was further investigated in the present study, and the results revealed that lung ADC cell lines exhibited downregulated expression levels of miR-429.

circRNAs have been widely recognized to function by interacting with miRNAs $(26,27)$. Certain circRNAs that interact with miRNA have been observed to be overexpressed in lung cancer. For example, hsa_circ_100395 affected the proliferation, migration and invasion of lung cancer cells by targeting the miR-1228/transcription factor 21 axis; this axis has been suggested to account for the lymph node metastasis, high TNM stage and low survival rate of patients with lung cancer (28). circ-PVT1 has been confirmed to interact with miR-124-3p and inversely regulate its expression to affect drug sensitivity in paclitaxel-resistant gastric cancer cells (7). The results of the present study revealed that circ-PVT1 could interact with miR-429 to regulate the functions of A549 cells.

The circRNA/miRNA/mRNA network plays a role in the mechanism through which circ-PVT1 affects the progression of lung ADC. The present results identified and validated the negative regulatory association between miR-429 and FOXK1 using a Pearson's correlation analysis and dual-luciferase reporter assays. FOXK1 has been reported to play a significant oncogenic role in various types of cancer, including esophageal, ovarian and colorectal cancer (29-31). In addition, FOXK1 expression levels have been found to be negatively associated with the overall survival of patients with esophageal cancer (29). The results of the present study revealed that the expression levels 
of FOXK1 were upregulated in lung ADC cells, while transfection with either the pcDNA3.1-FOXK1 plasmid or miR-429 inhibitor could abolish the inhibitory effects of circ-PVT1 knockdown on cell proliferation, migration and invasion. These results indicated that the knockdown of circ-PVT1 may inhibit the progression of lung ADC via the miR-429/FOXK1 signaling axis. Moreover, the current results demonstrated that the knockdown of circ-PVT1 enhanced the effect of DDP on the viability of A549/DDP cells, suggesting the promoting role of circ-PVT1 on the resistance of A549 cells to DDP. These findings are similar to the findings of a previous study, which reported that circ-PVT1, which was high expressed in doxorubicin- and DDP-resistant osteosarcoma cells, promoted the chemotherapy resistance of osteosarcoma cells (32).

In conclusion, the findings of the present study indicated that the knockdown of circ-PVT1 may inhibit the progression of lung ADC and enhance lung ADC cell sensitivity to DDP via the miR-429/FOXK1 signaling axis, which may provide potential novel targets for the treatment of lung ADC.

\section{Acknowledgements}

Not applicable.

\section{Funding}

The present study was funded by the General Program of National Natural Science Foundation of China (grant no. 81671905).

\section{Availability of data and materials}

The datasets used and/or analyzed during the current study are available from the corresponding author on reasonable request.

\section{Authors' contributions}

LC and DG conceived the study and performed the experiments. LC, XZ and XD analyzed and interpreted the data, and confirm the authenticity of all the raw data. LC wrote the manuscript. All authors read and approved the final manuscript.

\section{Ethics approval and consent to participate}

Not applicable.

\section{Patient consent for publication}

Not applicable.

\section{Competing interests}

The authors declare they have no competing interests.

\section{References}

1. Siegel RL, Miller KD and Jemal A: Cancer statistics, 2018. CA Cancer J Clin 68: 7-30, 2018.
2. Xu J, Zheng H, Yuan S, Zhou B, Zhao W, Pan Y and Qi D: Overexpression of ANLN in lung adenocarcinoma is associated with metastasis. Thorac Cancer 10: 1702-1709, 2019.

3. Tsutsui T, Yamaki H, Kumagai T, Omori C, Kobayashi H, Kakizaki Y and Miyashita Y: Small cell lung cancer with thyroid gland oligometastasis: A case report. Thorac Cancer 12: 387-390, 2021.

4. Denisenko TV, Budkevich IN and Zhivotovsky B: Cell death-based treatment of lung adenocarcinoma. Cell Death Dis 9: 117, 2018.

5. Eberle A, Jansen L, Castro F, Krilaviciute A, Luttmann S, Emrich K, Holleczek B, Nennecke A, Katalinic A, Brenner H and GEKID Survival Working Group: Lung cancer survival in Germany: A population-based analysis of 132,612 lung cancer patients. Lung Cancer 90: 528-533, 2015.

6. Qu S, Yang X, Li X, Wang J, Gao Y, Shang R, Sun W, Dou K and Li H: Circular RNA: A new star of noncoding RNAs. Cancer Lett 365: 141-148, 2015.

7. Liu YY, Zhang LY and Du WZ: Circular RNA circ-PVT1 contributes to paclitaxel resistance of gastric cancer cells through the regulation of ZEB1 expression by sponging miR-124-3p. Biosci Rep 39: BSR20193045, 2019.

8. Momen-Heravi $\mathrm{F}$ and Bala S: Emerging role of non-coding RNA in oral cancer. Cell Signal 42: 134-143, 2018.

9. Sun X, Luo L and Gao Y: Circular RNA PVT1 enhances cell proliferation but inhibits apoptosis through sponging microRNA-149 in epithelial ovarian cancer. J Obstet Gynaecol Res 46: 625-635, 2020.

10. Panda AC: Circular RNAs act as miRNA sponges. Adv Exp Med Biol 1087: 67-79, 2018

11. Zhu W, He J, Chen D, Zhang B, Xu L, Ma H, Liu X, Zhang Y and Le H: Expression of miR-29c, miR-93, and miR-429 as potential biomarkers for detection of early stage non-small lung cancer. PLoS One 9: e87780, 2014.

12. Xiao H, Zhu Q and Zhou J: Long non-coding RNA MALAT1 interaction with miR-429 regulates the proliferation and EMT of lung adenocarcinoma cells through RhoA. Int J Clin Exp Pathol 12: 419-430, 2019.

13. Zou J, Liu L, Wang Q, Yin F, Yang Z, Zhang W and Li L: Downregulation of miR-429 contributes to the development of drug resistance in epithelial ovarian cancer by targeting ZEB1. Am J Transl Res 9: 1357-1368, 2017.

14. Wang L, Mezencev R, Svajdler M, Benigno BB and McDonald JF: Ectopic over-expression of miR-429 induces mesenchymal-to-epithelial transition (MET) and increased drug sensitivity in metastasizing ovarian cancer cells. Gynecol Oncol 134: 96-103, 2014.

15. Sun H, Wang H, Wang X, Aoki Y, Wang X, Yang Y, Cheng X, Wang $Z$ and Wang $X$ : Aurora-A/SOX8/FOXK1 signaling axis promotes chemoresistance via suppression of cell senescence and induction of glucose metabolism in ovarian cancer organoids and cells. Theranostics 10: 6928-6945, 2020.

16. Long $Z$ and Wang Y: miR-195-5p suppresses lung cancer cell proliferation, migration, and invasion via FOXK1. Technol Cancer Res Treat 19: 1533033820922587, 2020.

17. Livak KJ and Schmittgen TD: Analysis of relative gene expression data using real-time quantitative PCR and the 2(-Delta Delta C(T)) method. Methods 25: 402-408, 2001.

18. Wang L, Liang Y, Mao Q, Xia W, Chen B, Shen H, Xu L, Jiang F and Dong G: Circular RNA circCRIM1 inhibits invasion and metastasis in lung adenocarcinoma through the microRNA (miR)-182/miR-93-leukemia inhibitory factor receptor pathway. Cancer Sci 110: 2960-2972, 2019.

19. Maass PG, Glazar P, Memczak S, Dittmar G, Hollfinger I, Schreyer L, Sauer AV, Toka O, Aiuti A, Luft FC and Rajewsky N: A map of human circular RNAs in clinically relevant tissues. J Mol Med (Berl) 95: 1179-1189, 2017.

20. Zhou R, Wu Y, Wang W, Su W, Liu Y, Wang Y, Fan C, Li X, $\mathrm{Li} \mathrm{G}$, Li Y, et al: Circular RNAs (circRNAs) in cancer. Cancer Lett 425: 134-142, 2018.

21. Wan L, Zhang L, Fan K, Cheng ZX, Sun QC and Wang JJ: Circular RNA-ITCH suppresses lung cancer proliferation via inhibiting the Wnt/ $\beta$-Catenin pathway. Biomed Res Int 2016: 1579490, 2016

22. Chen X, Mao R, Su W, Yang X, Geng Q, Guo C, Wang Z, Wang J, Kresty LA, Beer DG, et al: Circular RNA circHIPK3 modulates autophagy via MIR124-3p-STAT3-PRKAA/AMPKalpha signaling in STK11 mutant lung cancer. Autophagy 16: 659-671, 2020 . 
23. Ma X, Yang X, Bao W, Li S, Liang S, Sun Y, Zhao Y, Wang J and Zhao C: Circular RNA circMAN2B2 facilitates lung cancer cell proliferation and invasion via miR-1275/FOXK1 axis. Biochem Biophys Res Commun 498: 1009-1015, 2018.

24. Liu C, Hu W, Li LL, Wang YX, Zhou Q, Zhang F, Song-Yang YY, Zhu W, Sun CC and Li DJ: Roles of miR-200 family members in lung cancer: More than tumor suppressors. Future Oncol 14: 2875-2886, 2018

25. Xiao P, Liu W and Zhou H: miR-429 promotes the proliferation of non-small cell lung cancer cells via targeting DLC-1. Oncol Lett 12: 2163-2168, 2016.

26. Kulcheski FR, Christoff AP and Margis R: Circular RNAs are miRNA sponges and can be used as a new class of biomarker. J Biotechnol 238: 42-51, 2016.

27. Chen D, Ma W, Ke Z and Xie F: CircRNA hsa_circ_100395 regulates miR-1228/TCF21 pathway to inhibit lung cancer progression. Cell Cycle 17: 2080-2090, 2018.

28. Qu D, Yan B, Xin R and Ma T: A novel circular RNA hsa circ_0020123 exerts oncogenic properties through suppression of miR-144 in non-small cell lung cancer. Am J Cancer Res 8 : $1387-1402,2018$
29. Chen D, Wang K, Li X, Jiang M, Ni L, Xu B, Chu Y, Wang W, Wang H, Kang H, et al: FOXK1 plays an oncogenic role in the development of esophageal cancer. Biochem Biophys Res Commun 494: 88-94, 2017.

30. Li L, Gong M, Zhao Y, Zhao X and Li Q: FOXK1 facilitates cell proliferation through regulating the expression of $\mathrm{p} 21$, and promotes metastasis in ovarian cancer. Oncotarget 8 : 70441-70451, 2017.

31. Wu M, Wang J, Tang W, Zhan X, Li Y, Peng Y, Huang X, Bai Y, Zhao J, Li A, et al: FOXK1 interaction with FHL2 promotes proliferation, invasion and metastasis in colorectal cancer. Oncogenesis 5: e271, 2016.

32. Kun-Peng Z, Xiao-Long M and Chun-Lin Z: Overexpressed circPVT1, a potential new circular RNA biomarker, contributes to doxorubicin and cisplatin resistance of osteosarcoma cells by regulating ABCB1. Int J Biol Sci 14: 321-330, 2018.

cc) (i) $(-)$ This work is licensed under a Creative Commons

cc) $\mathrm{EY}$ NO ND Attribution-NonCommercial-NoDerivatives 4.0 International (CC BY-NC-ND 4.0) License. 